\title{
A Serosa-Originated Gastric Stromal Tumor Misdiagnosed by Ultrasonography and Frozen Section Pathology: A Case Report
}

This article was published in the following Dove Press journal: OncoTargets and Therapy

\author{
Lizhong Ren ${ }^{1} * *$ \\ Hongrong Qian ${ }^{1, *}$ \\ Junsen Wang ${ }^{2}$ \\ Piaopiao Jin $\mathbb{1}^{3}$ \\ Qida $\mathrm{Hu} \mathbb{1 D}^{4}$ \\ Jiajie $\mathrm{Yu}^{5}$ \\ Xin Zhang ${ }^{6}$ \\ Yun Zhang ${ }^{4}$ \\ Haifeng Huang ${ }^{5}$ \\ 'Department of Ultrasound, Shengzhou \\ People's Hospital, Shaoxing 312400, \\ People's Republic of China; ${ }^{2}$ Department \\ of Pathology, Shengzhou People's \\ Hospital, Shaoxing 3/2400, People's \\ Republic of China; ${ }^{3}$ Health Management \\ Center, First Affiliated Hospital, Zhejiang \\ University School of Medicine, Hangzhou \\ 310003, People's Republic of China; \\ ${ }^{4}$ Department of Hepatobiliary and \\ Pancreatic Surgery, First Affiliated \\ Hospital, Zhejiang University School of \\ Medicine, Hangzhou 310003, People's \\ Republic of China; ${ }^{5}$ Department of \\ Surgery, Shengzhou People's Hospital, \\ Shaoxing 312400, People's Republic of \\ China; ${ }^{6}$ Department of Radiology, \\ Shengzhou People's Hospital, Shaoxing \\ 312400, People's Republic of China \\ *These authors contributed equally to \\ this work
}

Correspondence: Junsen Wang Department of Pathology, Shengzhou People's Hospital, 666 Dangui Road, Shaoxing 3 2400 , People's Republic of China Tel +8657583338221

Email junsenwang@outlook.com

Haifeng Huang

Department of Surgery, Shengzhou People's Hospital, 666 Dangui Road, Shaoxing

312400, People's Republic of China

Tel +8657583338570

Email 18241425@qq.com

\begin{abstract}
Gastric gastrointestinal stromal tumor (GIST) is a mesenchymal neoplasm, typically arising from the muscularis propria layer of the stomach wall. Serosa-derived GIST is rarely seen and has not been reported yet. A 49-year-old Chinese female was presented with marked abdominal distension. Ultrasonography revealed a retroperitoneal tumor adjacent to the stomach wall with an intact structure of five echo layers, indicating a non-stomach tumor origin. Preoperative radiological studies suggested tight tumor-stomach adjacency, which was confirmed by intraoperative dissection. Initial frozen section indicated a pathological diagnosis of spindle cell tumor, which turned out to be a gastric GIST originated from the serosa layer of the stomach wall. The current case demonstrates the rare occurrence of serosa-derived GIST. This case also suggests difficulties in preoperative diagnosis of gastric GISTs, especially when uncommon pathological conditions like rare tumor origins were presented.
\end{abstract}

Keywords: gastric gastrointestinal stromal tumor, serosa layer, schwannoma, misdiagnosis

\section{Introduction}

Gastric gastrointestinal stromal tumors (GIST) are mesenchymal neoplasms with a population-based annual incidence rate of 0.38 cases per $100,000 .{ }^{1}$ In $90 \%$ of the GISTs, mutations of KIT or, less commonly, PDGFRA are activated and implicated in the tumorigenesis. ${ }^{2,3}$ GISTs are therefore positive for KIT (CD117), the majority of which also express DOG1 and CD34. ${ }^{4}$ However, definitive diagnosis before histopathology analysis is difficult for gastric GISTs, because there are other types of mesenchymal tumors in the gastrointestinal tract that can partially overlap with GISTs in gross characteristics and histopathology. ${ }^{5}$

Gastric GISTs typically arise from the muscularis propria layer of the stomach wall, rarely also from the submucosa layer due to its stromal nature. ${ }^{6}$ Under normal condition, five echo layers, namely, superficial mucosa, muscularis mucosa, submucosa, muscularis proper, and serosa, could be demonstrated by ultrasonography. ${ }^{7}$ Involvement of the echo layers might help determine local infiltration of GIST in the stomach wall. We recently treated a gastric GIST with intact five layers of stomach wall between the gastric cavity and the tumor, which turned out to be a serosa-derived GIST. Herein, we presented this rare GIST case perioperatively misdiagnosed by ultrasonography and frozen section pathology. 


\section{Case Presentation}

A 49-year-old female Chinese patient was presented to our hospital with upper abdominal distension for 3 months. Marked abdominal distension had been observed more frequently for 10 days. Physical examination and lab test results were unremarkable.

Routine ultrasonography revealed a retroperitoneal tumor of $5.0 \times 3.7 \times 4.1 \mathrm{~cm}$ with a clear edge, located between stomach and pancreas and adjacent to the posterior stomach surface. Notably, gastric tissue adjacent to the tumor showed an intact structure of five layers of the stomach wall (Figure 1). And the diagnosis from the ultrasonographist was a benign tumor, possibly schwannoma, derived from retroperitoneum or mesentera rather than from stomach.

On the contrary, a contrasted computed tomography (CT) was unable to rule out that the tumor was originated from the stomach wall due to tight tumor-stomach adjacency (Figure 2). Further magnetic resonance imaging (MRI) suggested that the tumor was close to the stomach wall with gastric mucosa uninvolved (Figure $3)$. We also observed uniformly progressive enhancement with several low-density areas inside the tumor. The radiologist suggested a diagnosis of gastrointestinal mesenchymal tumor but preferred stromal tumor rather than schwannoma.

The tumor board, therefore, suggested laparoscopic radical resection of the peritoneal tumor. Intraoperative dissection showed the tumor was strongly adjacent to the posterior stomach wall and free from the retroperitoneum. The initial frozen section indicated a pathological diagnosis of spindle cell tumor, which might be gastric schwannoma. However, the final pathology via paraffin section showed that the tumor is a gastric stromal tumor originated from the serosa layer of the stomach wall, with a mitotic rate of over 5 per 50 high power field. Immunohistochemistry results also supported the characteristic marker expression as gastric stromal tumor, including positive CD117, positive DOG-1, negative S100, 5\% positive Ki67, negative SMA, negative CKpan, $8 \%$ positive p53, and positive CD34 (Figure 4). The gastric stromal tumor was at stage IIIA with a high risk of recurrence, according to the 8th AJCC staging system. $^{8}$

The patient recovered well from the surgery. The tumor specimen was sent for mutation analysis. A substitution mutation in KIT exon 11, NM_000222:
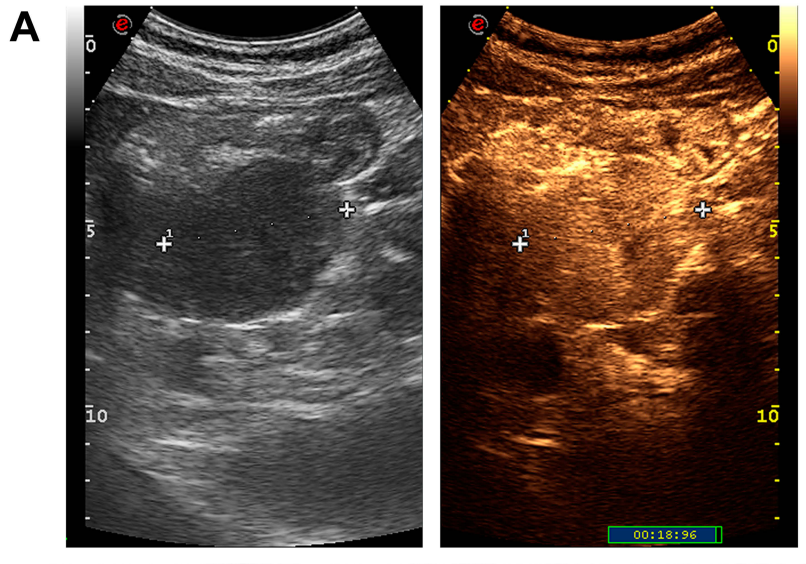

B

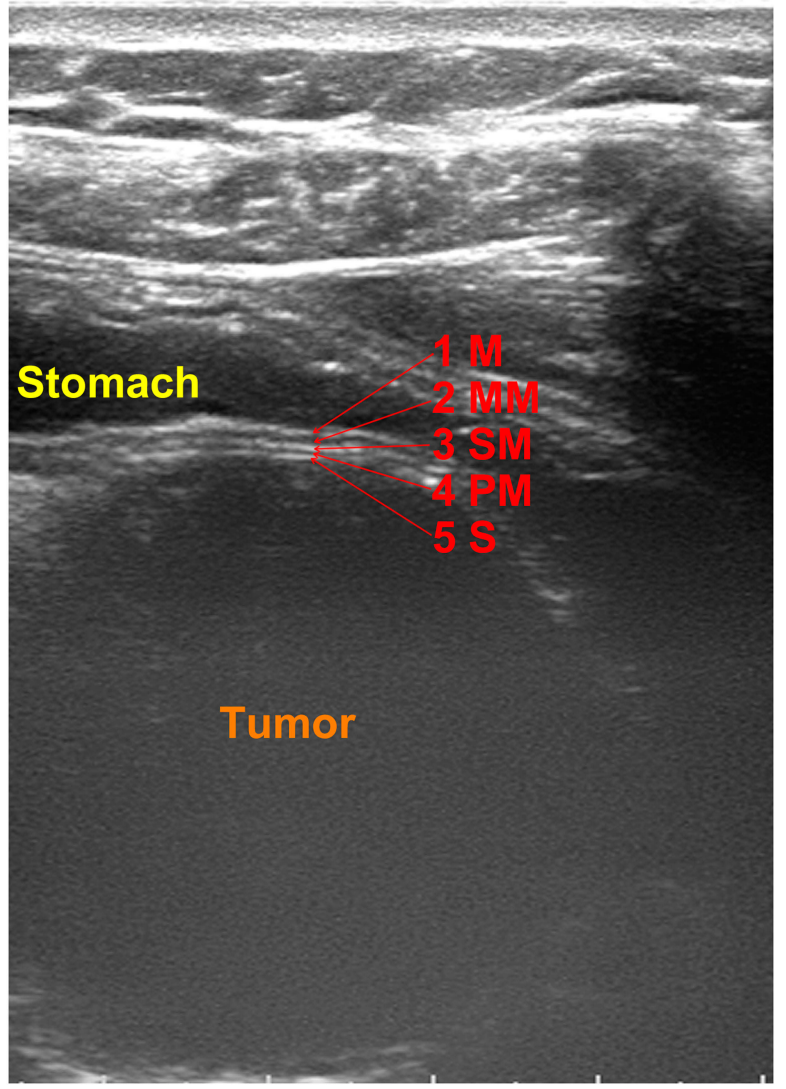

Figure I Ultrasound demonstration of the tumor. (A) Contrast-enhanced ultrasound image with size measurement as $5.0 \mathrm{~cm}$ at $19 \mathrm{~s}$ post contrast administration. (B) The relationship of the stomach and the tumor. Intact five layers of the stomach wall between the stomach and the tumor were indicated as: I $M$ - superficial mucosal layer (hyperechoic), 2 MM - muscularis mucosal layer (hypoechoic), 3 SM submucosal layer (hyperechoic), 4 PM - muscularis proper layer (hypoechoic), and 5 $\mathrm{S}$ - serosa (hyperechoic).

c.1676T $>$ A (p.V559D), was detected, but no other mutation in the KIT or PDGFRA gene was presented. The patient was then scheduled with the treatment of adjuvant imatinib $400 \mathrm{mg}$ per day for the following 3 years to reduce recurrence. She has been free of recurrence during the 6-month follow-up period since surgical resection. 

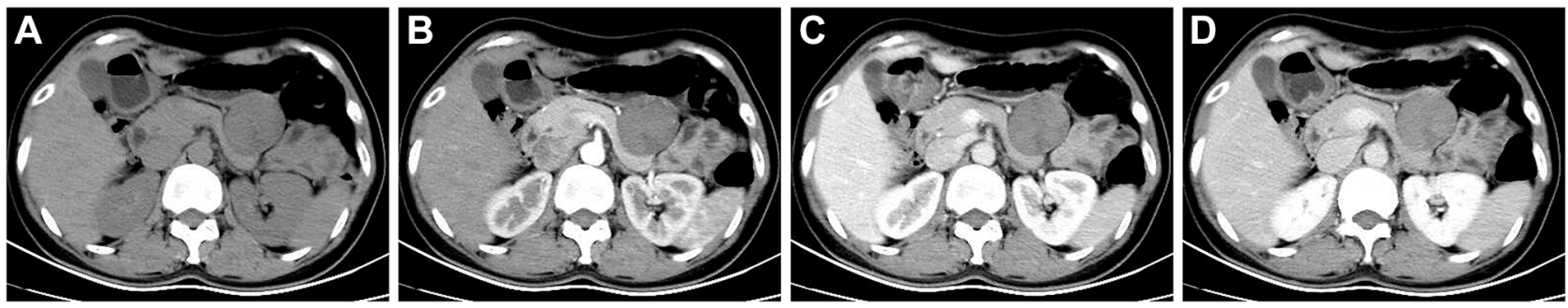

Figure 2 Preoperative CT investigation with $(\mathbf{A})$ non-enhanced, $(\mathbf{B})$ arterial phase, $(\mathbf{C})$ venous phase, and (D) delayed phase images.
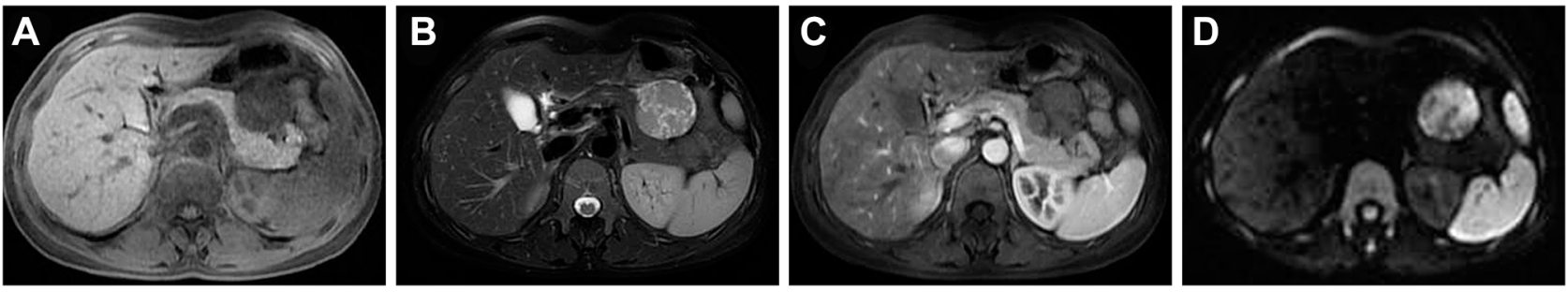

Figure 3 Preoperative MR investigation with (A) T1 weighted, (B) T2 weighted, (C) arterial phase images, and (D) diffusion-weighted imaging.

\section{Discussions}

The current case features difficulties in preoperatively obtaining a definitive diagnosis for gastric stromal tumor derived from the gastric serosa layers. Two different diagnoses of the tumor type (stromal tumor or schwannoma) and the pathological origin (gastric or retroperitoneal) were proposed, based on different diagnostic approaches. Retrospective review after surgical resection showed that the results of ultrasonography and frozen section pathology were not consistent with the final diagnosis.

The most confusing result is the ultrasound presentation of the stomach wall between the gastric cavity and the tumor, with the intact five layers. The rare misdiagnosis could occur when the stromal tumors are derived from serosa or subserosa, because serosa layer and the tumor margin might present similar echo intensity. As stromal tumors originate from CD34-positive interstitial cells of Cajal (ICCs) within the wall of digestive system, ${ }^{9,10}$ but subserosal ICCs were not frequently seen, ${ }^{11,12}$ indicating the rareness of the serosal or subserosal origin of the stromal tumors. Meanwhile, the misdiagnosis by ultrasonography also suggests a minority of stromal tumors occur without invasion of the mucosa, submucosa, muscularis externa or even subserosa layer.

Differential diagnosis between schwannoma diagnosed by frozen section pathology and stromal tumor is often difficult. Gastric schwannoma is a rare mesenchymal tumor characterized by excessive proliferation of Schwann cells, consisting of $6.3 \%$ of all the gastrointestinal mesenchymal tumors. ${ }^{5,13}$ Histologically, schwannomas could present with interlacing bundles of spindle cells and nuclear palisading. ${ }^{13}$ On the contrary, nuclear palisading is reported in $33 \%$ of gastric stromal tumors. ${ }^{14}$ Therefore, schwannomas share similarity with stromal tumors which causes misdiagnosis in frozen section pathology analysis. However, schwannomas often stain positive for S100 and negative for CD117, DOG-1, and CD34, which is opposite to the results in gastric stromal tumors. ${ }^{13,15}$ Above all, accurate distinction between schwannomas and stromal tumors rests on immunophenotyping, as cytologic studies like frozen section study cannot separate stromal tumors from schwannomas.

In conclusion, we reported a gastric stromal tumor case derived from serosa layer, which was misdiagnosed by ultrasonography and froze section pathology. The current case suggests difficulties in preoperative diagnosis of gastric stromal tumors, especially when uncommon pathological conditions like rare tumor origins were presented.

\section{Ethics Approval and Consent for Publication}

This study was approved by the research ethics committee of Shengzhou People's Hospital, and written informed consent for publication of the clinical details and images was obtained from the patient. 

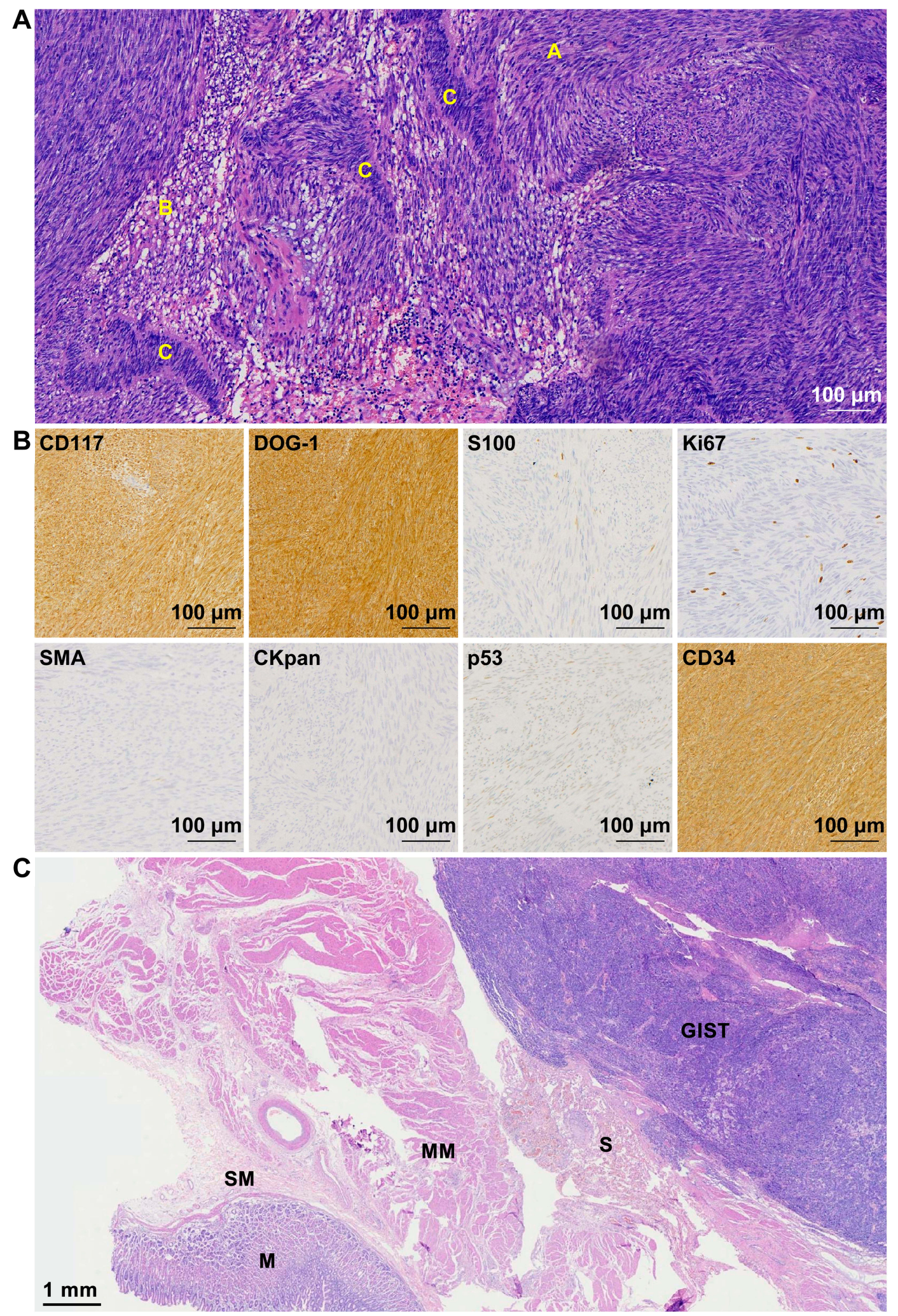

Figure 4 Results of pathology analysis. (A) An overview of histology by H\&E stain. Zone A indicates the area composed of abundant spindle cells. Zone B indicates the area with loose structure and paranuclear vacuolization. Zone $C$ indicates spindles cells with nuclear arrangement of the palisades. (B) Immunohistochemistry analyses showing expression of CDII7, DOG-I, SI00, Ki67, SMA, CKpan, P53, and CD34. (C) Pathological analysis of the relationship between the gastric wall layers and the GIST tumor. 


\section{Informed Consent Statement}

Informed written consent was obtained from the patient for publication of this report and any accompanying images.

\section{Acknowledgments}

We thank the hepatobiliary and pancreatic multidisciplinary board of Shengzhou People's Hospital for the assistance and helpful advice.

\section{Author Contributions}

Ren L and Qian H performed ultrasonography, reviewed the related literature, and contributed equally to this work; Wang $\mathrm{J}$ performed the pathological analyses and interpretation; Jin $\mathrm{P}$ and $\mathrm{Hu} \mathrm{Q}$ collected the data, and drafted the manuscript; $\mathrm{Hu}$ Q, Yu J, Huang H, and Zhang Y were the patients' surgeons, performed the operation, and analyzed the data; Zhang $\mathrm{X}$ analyzed and interpreted the imaging findings; Wang $\mathrm{J}$, Huang $\mathrm{H}$, and Zhang $\mathrm{Y}$ were responsible for the revision of the manuscript for important intellectual content; all authors then contributed to data analysis, drafting and revising the article, gave final approval of the version to be published, and agreed to be accountable for all aspects of the work.

\section{Funding}

This work was financially supported by the National Natural Science Foundation of China (Nos. 81972207, 81830089, 81537009,81502026 , and 51573161), the Zhejiang Natural Science Foundation (Nos. LQ16H18000245 and LY18H16 0026), and the Shengzhou Technology Program (No. 201909).

\section{Disclosure}

The authors report no conflicts of interest in this work.

\section{References}

1. Ma GL, Murphy JD, Martinez ME, Sicklick JK. Epidemiology of gastrointestinal stromal tumors in the era of histology codes: results of a population-based study. Cancer Epidemiol Biomarkers Prev. 2015;24:298-302. doi:10.1158/1055-9965.EPI-14-1002

OncoTargets and Therapy

\section{Publish your work in this journal}

OncoTargets and Therapy is an international, peer-reviewed, open access journal focusing on the pathological basis of all cancers, potential targets for therapy and treatment protocols employed to improve the management of cancer patients. The journal also focuses on the impact of management programs and new therapeutic
2. Hemming ML, Heinrich MC, Bauer S, George S. Translational insights into gastrointestinal stromal tumor and current clinical advances. Ann Oncol. 2018;29:2037-2045. doi:10.1093/annonc/mdy309

3. Wada R, Arai H, Kure S, Peng WX, Naito Z. "Wild type" GIST: clinicopathological features and clinical practice. Pathol Int. 2016;66:431-437. doi:10.1111/pin.12431

4. Nishida T, Blay JY, Hirota S, Kitagawa Y, Kang YK. The standard diagnosis, treatment, and follow-up of gastrointestinal stromal tumors based on guidelines. Gastric Cancer. 2016;19:3-14. doi:10.1007/ s10120-015-0526-8

5. Abraham SC. Distinguishing gastrointestinal stromal tumors from their mimics: an update. Adv Anat Pathol. 2007;14:178-188. doi:10.1097/PAP.0b013e318050aa66

6. Scherubl H, Faiss S, Knoefel WT, Wardelmann E. Management of early asymptomatic gastrointestinal stromal tumors of the stomach. World J Gastrointest Endosc. 2014;6:266-271. doi:10.4253/wjge.v6. i7.266

7. Gale HI, Gee MS, Westra SJ, Nimkin K. Abdominal ultrasonography of the pediatric gastrointestinal tract. World J Radiol. 2016;8: 656-667. doi:10.4329/wjr.v8.i7.656

8. DeMatteo RP, Maki RG, Agulnik M, et al. Gastrointestinal stromal tumor. In: Amin MB, editor. AJCC Cancer Staging Manual, 8th. Chicago: American Joint Committee on Cancer; 2017:523-529.

9. Wang L, Vargas H, French SW. Cellular origin of gastrointestinal stromal tumors: a study of 27 cases. Arch Pathol Lab Med. 2000;124:1471-1475. doi:10.1043/0003-9985(2000)124<1471:COO GST $>2.0 . \mathrm{CO} ; 2$

10. Sircar K, Hewlett BR, Huizinga JD, Chorneyko K, Berezin I, Riddell RH. Interstitial cells of Cajal as precursors of gastrointestinal stromal tumors. Am J Surg Pathol. 1999;23:377-389. doi:10.1097/ 00000478-199904000-00002

11. Miettinen M, Lasota J. Gastrointestinal stromal tumors: review on morphology, molecular pathology, prognosis, and differential diagnosis. Arch Pathol Lab Med. 2006;130:1466-1478. doi:10.10 43/1543-2165(2006)130[1466:GSTROM]2.0.CO;2

12. Komuro T. Structure and organization of interstitial cells of Cajal in the gastrointestinal tract. J Physiol. 2006;576:653-658. doi:10.1113/ jphysiol.2006.116624

13. Hou YY, Tan YS, Xu JF, et al. Schwannoma of the gastrointestinal tract: a clinicopathological, immunohistochemical and ultrastructural study of 33 cases. Histopathology. 2006;48:536-545. doi:10.1111/ j.1365-2559.2006.02370.x

14. Miettinen M, Sobin LH, Lasota J. Gastrointestinal stromal tumors of the stomach: a clinicopathologic, immunohistochemical, and molecular genetic study of 1765 cases with long-term follow-up. Am $J$ Surg Pathol. 2005;29:52-68. doi:10.1097/01.pas.0000146010. 92933.de

15. Gulati R, Hanson JA, Parasher G, Castresana D. Getting the gist of a schwannoma. Gastrointest Endosc. 2020;91:191-192. doi:10.1016/j. gie.2019.07.027 agents and protocols on patient perspectives such as quality of life, adherence and satisfaction. The manuscript management system is completely online and includes a very quick and fair peer-review system, which is all easy to use. Visit http://www.dovepress.com/ testimonials.php to read real quotes from published authors. 